\title{
Clinical Factors Associated with Inadequate Treatment Response to Methotrexate among Patients with Rheumatoid Arthritis in a Tertiary Referral Hospital
}

\author{
Grace G. Penserga, MD and Ester G. Penserga, MD \\ Division of Rheumatology, Department of Medicine, Philippine General Hospital, University of the Philippines Manila
}

\begin{abstract}
Objective. The study aimed to determine patient-related factors associated with inadequate treatment response of patients with rheumatoid arthritis (RA) to methotrexate (MTX).

Methodology. We reviewed the medical records of patients with RA seen at the rheumatology outpatient clinic of Philippine General Hospital; dichotomized into those with adequate and inadequate treatment response to MTX. Clinical data including age, gender, comorbidities, smoking status, tender joint counts, disease activity score-28 (DAS28), and rheumatoid factor (RF) positivity were compared between the two groups. We used univariate binary logistic regression to determine whether these clinical factors were significantly associated with inadequate treatment response.
\end{abstract}

Results. Majority (47/77 or 61\%) of patients have inadequate treatment response to MTX. There were no significant differences between the two study groups in terms of age, tender joint count, DAS28, sex, smoking status and RF positivity. These factors also failed to show significant association with inadequate treatment response.

Conclusion. Age, sex, smoking status, duration of disease, initial DAS28, RF positivity and tender joint counts are not significantly associated with inadequate treatment response. A larger sample size is needed to estimate the prevalence of inadequate treatment response to MTX and derive a predictive model for treatment response.

Key Words: methotrexate, rheumatoid arthritis

Corresponding author: Grace G. Penserga, MD

Division of Rheumatology

Department of Medicine

Philippine General Hospital, University of the Philippines Manila

Taft Avenue, Manila 1000, Philippines

Email: ggpenserga@up.edu.ph

\section{INTRODUCTION}

Rheumatoid arthritis (RA) is a chronic, multisystemic autoimmune disease that affects individuals in the fourth and fifth decade of life. ${ }^{1,2}$ At the onset, RA causes joint swelling, pain and limitation of motion, and if left untreated, leads to severe joint destruction, deformities and disability. ${ }^{3}$ Early intervention and achievement of low disease activity or disease remission are therefore the treatment goals of current practice guidelines for RA. ${ }^{1}$

Methotrexate (MTX) is a conventional disease-modifying anti-rheumatic drug (cDMARD) that has antiinflammatory and anti-proliferative effects mediated by adenosine release. ${ }^{4}$ It is considered as first-line and cornerstone therapy for RA due to its efficacy, safety and low cost. However, studies show that as high as $30 \%$ to $50 \%$ of patients do not achieve therapeutic targets and eventually require additional agents. ${ }^{4-9}$ Adjunct treatment options include another cDMARD like hydroxychloroquine, 
or a biologic DMARD (bDMARD) such as tumor necrosis factor inhibitors or small-molecule inhibitors. ${ }^{1}$ Adding these agents to the treatment regimen early in the disease course, or when inadequate to response to methotrexate is recognized, is a predictor of good outcomes for patients with RA. ${ }^{5}$

Several studies have attempted to identify clinical factors that predispose individuals with RA to inadequate treatment response to methotrexate. A high disease activity index at baseline was associated with insufficient response in several studies, as well as female sex, a higher joint count and longer treatment delay. ${ }^{7,10}$ Smoking status and rheumatoid factor positivity also predicted inadequate response to therapy. ${ }^{4-11}$

In the Philippines, MTX is the most commonly prescribed drug for individuals with RA. ${ }^{2}$ However, local data on the prevalence and predictors of inadequate treatment response is lacking. The objective of the study was to determine whether patient characteristics identified in literature review such as age, sex, disease activity score-28 (DAS28), delay to treatment, smoking status and RF positivity are associated with inadequate treatment response to methotrexate.

\section{MATERIAL AND METHODS}

\section{Study design, setting and population}

We conducted a medical records review of patients seen at the rheumatology outpatient clinic of PGH based on the following inclusion criteria: 1) diagnosed with RA based on the 2010 American College of Rheumatology Classification Criteria, ${ }^{12} 2$ ) maintained on methotrexate monotherapy for at least 6 months, with addition of non-steroidal antiinflammatory drugs (NSAIDs) and/or corticosteroids as deemed necessary, and 3) age of at least 19 years. We excluded if 1) they were given combination conventional DMARD or biologic DMARDs after less than 6 months of methotrexate therapy, 2) they were poorly compliant with methotrexate therapy, and 3) had incomplete medical records or lacked data relevant to the objective of the study. Poor compliance was defined as having missed MTX doses for 2 consecutive weeks

\section{Sample size calculation}

At 95\% confidence level and 5\% confidence limits, the minimum required sample size was 81 , assuming that the prevalence of inadequate treatment response is $50 \%$.

\section{Data Collection}

Demographic data collected included gender, comorbidities and smoking status. Clinical and RA-related data collected were tender joint counts, DAS28 score and RF status. Patients were then divided into two groups: those with adequate treatment response, defined as the achievement of low disease activity or disease remission after 6 months, and those with inadequate treatment response to MTX. The clinical data of these two groups were compared.

\section{Statistical Analysis}

Normal data were presented in means with standard deviations and proportions. T-test was used to test the differences in mean values. Chi-squared test was used to compare proportions between the two groups. Risk factor analysis was performed using logistic regression, where a P-value of less than 0.05 was considered statistically significant.

\section{Ethical considerations}

The protocol was approved by the site's ethical review board prior to data collection.

\section{RESULTS}

Out of 120 patients screened, 77 patients with RA were included in the study. The average age of patients was 52.6 years. Only $7.8 \%$ are male, while $79.2 \%$ were married and $53.2 \%$ were ever employed. The most common comorbidities were hypertension (41.6\%), diabetes mellitus (15.6\%) and stroke (7.8\%). Majority (90.9\%) were non-smokers and non-alcoholic beverage drinkers (90.9\%). Seventy-four percent were $\mathrm{RF}$ positive. The mean time to therapy was 21.4 months. (Table 1 )

Only $7 \%$ of the patients had one joint involvement at presentation; most had greater than three joints (70.1\%). After 6 months of methotrexate therapy, there was decrease in average tender joint count (3.4, from 9.6 at baseline), mean ESR (43.2, from 53.3), and mean DAS28 (4.6, from 5.2). Around $46.8 \%$ of the patients were classified as high in disease activity prior to treatment, while $48.1 \%$ were still classified as moderate after MTX therapy. Those with high disease activity at baseline appear to have benefitted more from treatment than those with moderate disease activity. Nevertheless, majority (61\%) had inadequate treatment response to MTX. (Table 2)

There were no significant differences in the mean age, baseline tender joint count, baseline DAS 28, RF positivity and disease duration prior to MTX treatment between the those with adequate versus inadequate response to MTX. There was no association between these clinical factors to the risk for inadequate treatment response to MTX. (Table 3)

\section{DISCUSSION}

Rheumatoid arthritis is a chronic autoimmune disease that causes severe joint damage, deformity and disability, usually affecting individuals in their fourth to fifth decade of life. Data from the Philippine Rheumatoid Arthritis Database and Registry (RADAR) show that majority of Filipino RA patients are female, employed, and had disease onset at 49.44 years old. The lag time from disease onset to diagnosis was 5.4 years. The mean BMI was 23.83, and most denied ever smoking cigarettes. Most patients presented with polyarticular disease, involving the wrist, 
Table 1. Demographic profile of the study population $(N=77)$

\begin{tabular}{lc}
\hline \multicolumn{1}{c}{ Characteristics } & No. (\%) \\
\hline Age (years), Mean \pm SD & $52.6 \pm 11.0$ \\
\hline Sex (Male) & $6(8)$ \\
\hline Civil Status (Married) & $61(79)$ \\
\hline Employment (Ever) & $41(53)$ \\
\hline Previously diagnosed medical conditions & $32(42)$ \\
Hypertension & $12(16)$ \\
Diabetes mellitus & $6(8)$ \\
Stroke & $5(7)$ \\
Tuberculosis & $4(5)$ \\
Kidney disease & $3(4)$ \\
Bronchial asthma & $3(4)$ \\
Thyroid disorders & $2(3)$ \\
Coronary artery disease & $1(1)$ \\
Dyslipidemia & $1(1)$ \\
Osteoarthritis & $1(1)$ \\
Secondary Cushing syndrome & \\
\hline Smoking status & $70(91)$ \\
Non-smoker & $4(5)$ \\
Current smoker & $3(3)$ \\
Quit Smoking & $70(91)$ \\
\hline Alcohol drinking & $6(8)$ \\
Non-alcohol drinker & $1(1)$ \\
Social drinker & $0(0)$ \\
Mild to moderate & 57 (74) \\
\hline Heavy & \\
\hline Time-positive & \\
\hline$R F$, Rheumatoid factor; DMARD, Disease-modifying anti-rheumatic drugs
\end{tabular}

Table 2. Comparison of disease activity prior to and after starting MTX

Characteristics

Prior to MTX After MTX

\begin{tabular}{lcc} 
Tender joint count, Mean \pm SD & $9.6 \pm 9.5$ & $3.4 \pm 7.4$ \\
\hline ESR, Mean \pm SD & $53.3 \pm 29.2$ & $43.2 \pm 25.7$ \\
\hline DAS28, Mean \pm SD & $5.2 \pm 1.3$ & $4.6 \pm 5.4$ \\
\hline Disease Activity & & \\
$\quad$ High & $36(47)$ & $14(18)$ \\
Moderate & $38(49)$ & $37(48)$ \\
Low & $3(4)$ & $21(27)$ \\
$\quad$ Remission & $0(0.0)$ & $5(7)$ \\
\hline Treatment response & & \\
$\quad$ Inadequate & &
\end{tabular}

metacarpophalangeal joints and proximal interphalangeal joints; average tender joint count of 14; and average DAS28 of 5.27 that translates to high disease activity. Methotrexate is the most commonly prescribed synthetic disease-modifying anti-rheumatic drug (sDMARD) with an average dose of $8.6 \mathrm{mg} / \mathrm{week}$, followed by hydroxychloroquine. Follow-up data show that mean DAS28 after 3-6 months is 4.59, 4.52 at 1 year and 4.45 at 3 years. $^{2}$ These disease activity scores translate to moderate disease activity, which falls short of the therapeutic targets of low disease activity or disease remission.

Compared to patients in the RADAR study, RA patients included in this study followed the clinical profile of RA patients enrolled in RADAR in terms of age, gender, comorbidities, smoking status and RF positivity. A key difference is that the lag time from disease onset to initiation of MTX therapy is much shorter in this study (1.7 years) compared to that in the RADAR study $\left(5.4\right.$ years). ${ }^{2}$ This

Table 3. Association of factors with outcome of inadequate response to MTX

Risk Factors for Inadequate Response

Age (years) (Mean \pm SD)

Disease activity prior to MTX: Tender joint count

Disease activity Prior to MTX: DAS28

Time to DMARD (months) (Mean \pm SD)

Presence of comorbid disease

\begin{tabular}{|c|c|c|c|c|c|}
\hline Yes & $19(63)$ & $26(55)$ & \multicolumn{3}{|c|}{ Reference } \\
\hline No & $11(37)$ & $21(45)$ & 0.72 & 0.28 to 1.83 & 0.49 \\
\hline \multicolumn{6}{|l|}{ Smoking status } \\
\hline Non-smoker & $28(93)$ & $42(89)$ & \multicolumn{3}{|c|}{ Reference } \\
\hline Ever smoker & $2(7)$ & $5(1)$ & 1.67 & 0.30 to 9.20 & 0.56 \\
\hline \multicolumn{6}{|l|}{ Sex } \\
\hline Male & $3(10.0)$ & $3(6.4)$ & \multicolumn{3}{|c|}{ Reference } \\
\hline Female & $27(90.0)$ & $47(93.6)$ & 0.61 & 0.12 to 3.26 & 0.57 \\
\hline \multicolumn{6}{|c|}{ Rheumatoid factor } \\
\hline Negative & $9(30.0)$ & $11(23.4)$ & \multicolumn{3}{|c|}{ Reference } \\
\hline Positive & $21(70.0)$ & $36(76.6)$ & 1.40 & 0.50 to 3.94 & 0.52 \\
\hline
\end{tabular}

Adequate Response $(n=30) \quad$ Inadequate Response $(n=47) \quad$ OR $\quad 95 \% \mathrm{Cl} \quad \mathrm{p}$ value

\begin{tabular}{ccccc}
$52.1 \pm 10.4$ & $53.0 \pm 11.5$ & 1.01 & 0.97 to 1.05 & 0.71 \\
\hline $9.6 \pm 11.6$ & $9.6 \pm 8.1$ & 1.00 & 0.95 to 1.05 & 0.99 \\
\hline $5.1 \pm 1.2$ & $5.4 \pm 1.4$ & 1.20 & 0.83 to 1.72 & 0.33 \\
\hline $21.1 \pm 19.4$ & $21.5 \pm 20.4$ & 1.00 & 0.98 to 1.02 & 0.94 \\
\hline
\end{tabular}


Table 4. Comparison of disease activity prior to and after starting MTX

Characteristics

RADAR Study Population

Time to DMARD therapy, years

5.40

1.7

DAS28 prior to MTX, Mean \pm SD

5.27

5.2

DAS28 prior to MTX, Mean \pm SD

4.59

4.6

MTX, Methotrexate; DMARD, Disease-modifying anti-rheumatic drugs

may reflect an overall improvement in the timely diagnosis and management of RA patients. The mean tender joint count seen in this study prior to MTX therapy lower than those seen in RADAR (14 versus 9.6), but the mean DAS28 prior to initiation of MTX and after 6 months of treatment were comparable. (Table 4)

To prevent morbidity and disability, local and international guidelines have uniformly pushed for treating RA early in its course, and treating to target low disease activity or remission. ${ }^{1}$ Methotrexate remains the firstline and anchor drug for its efficacy, safety and low cost, but studies show that inadequate treatment response to MTX is prevalent (30\%-50\%)., ${ }^{5,911}$ For this study, $61 \%$ had inadequate treatment response to MTX. The emergence of biologic DMARDs in the past decade have greatly expanded treatment options beyond methotrexate. ${ }^{5,6}$ We recognize the need to diagnose patients as poor responders to methotrexate and manage accordingly.

Several studies have attempted to identify certain clinical factors that can predict an inadequate response to MTX. A high DAS28 at presentation had been identified as a possible predictor of poor response. The U-Act-Early, a 2-year multicenter, randomized, double-blind placebo-controlled trial that evaluated 108 DMARD-naive patients with RA showed an initial DAS28 with an adjusted odds ratio of 2.1 (95\% CI 1.4 to 3.2) for an inadequate response. ${ }^{7}$ Another multicenter single-blinded trial of $285 \mathrm{RA}$ patients led by De Rotte, et al, also found that a DAS28 of greater than 5.1 is a strong predictor for inadequate treatment response. ${ }^{11} \mathrm{In}$ contrast, our study showed that the initial DAS28 does not correlate with poor treatment response (OR 1.2, 95\% CI 0.83-1.72).

Anderson et al, which analyzed primary data from 1435 patients enrolled in 14 diverse clinical trials, identified female sex to have reduced likelihood of treatment response (OR 0.79 , p-value 0.05$).{ }^{10}$ Similarly, the systematic review of 11 RCTs and 2 prospective studies by Drouin, et al, found that male sex and a low DAS28, are the strongest determining factors of a good clinical response to MTX. ${ }^{9}$ In contrast, the U-Act-Early study did not find a significant association between female sex and poor treatment response. This is consistent with the results of our study, in which female sex only confers an OR of 0.61 (95\% CI 0.12, 3.26).

The multicenter observational study of Sargeant, et al. determined that a longer symptom duration predicts an inadequate treatment response (OR 1.00, 95\% CI 1.00,
1.00). ${ }^{8}$ In contrast, our study shows that symptom duration prior to initiation of MTX does not significantly correlate with inadequate treatment response (OR 1.00, 95\% CI $0.97,1.02)$

Among other risk factors, current smoking status was included in the prediction model developed by De Rotte, et al. ${ }^{11}$ Two other separate study groups also identified smoking as a risk factor for poor treatment response: Sargeant et al. (OR 1.78, 95\% CI 1.28, 2.48), and Teitsma et al. (OR 3.02, $95 \%$ CI 1.1, 8.0) for current smoking status, and that the effect of smoking on the risk of inadequate response is dosedependent. ${ }^{7,8}$ Our study, however, did not find a significant correlation between cigarette smoking and inadequate treatment response. RF positivity did not correlate significantly with poor treatment response: not being RFpositive had an OR of 0.6 with 95\% CI 0.50, 0.88, (Sargeant et al.); and RF positivity had an OR of 1.30 with $95 \%$ CI 0.50, 3.37 (Teitsma et al). ${ }^{7,8}$ Consistent with these findings, our study does not find a significant association between $\mathrm{RF}$ positivity and poor treatment response (OR 1.4, 95\% CI $0.50,3.94$ ).

Closely related to DAS28 is the number of tender joints at presentation. The prospective observational study led by Sergeant et al. identified a higher tender joint count (OR $1.06,95 \%$ CI 1.02,1.10) per additional tender joint to be independent predictors of MTX non-response. ${ }^{8}$ However, Drouin et al. noted that the number of tender joints is not significantly associated with inadequate response to treatment. ${ }^{9}$ In our study, tender joint counts also did not confer risk for poor treatment response (OR 1.00, 95\% CI $0.95,1.05)$.

There are several limitations in this study. The study did not reach the target sample size, and is underpowered for the correlational analysis. We did not include in the analysis the presence of anti-CCP antibody, a more specific antibody to RA that also portends a poorer prognosis. This clinical factor was not uniformly obtained from all patients and was thus excluded. As this was a retrospective study, the escalation of methotrexate and background prednisone doses, route of MTX administration, and co-administration with prednisone or NSAIDS within the first 6 months of treatment largely depended on the clinician's judgement at the time of examination. This resulted in a non-uniform dosing of MTX and other anti-inflammatory medications among the study population; a higher or lower dose of MTX and background anti-inflammatory medications would have affected the rate at which the target disease activity was reached. It should be noted, however, that one of the primary assumptions of the study was that all patients were managed as per the 2015 American College of Rheumatology guideline for the treatment of RA, which recommended that regardless of baseline disease activity, a treat-to-target approach was recommended over non-targeted approach. No specific dose or dose escalation was recommended, so long as the target is reached. Closely related to this, an assumption 
was also made that disease flares were managed as per guideline: prednisone can be added at the lowest possible dose and the shortest possible duration. Though no specific dose and duration was stated, the guideline recommends a dose of no more than $10 \mathrm{mg} /$ day for less than 3 months. ${ }^{1}$

\section{CONCLUSION}

Majority of patients did not reach treatment targets on methotrexate. Age, sex, smoking status, duration of disease, initial DAS 28, RF positivity and tender joint counts failed to show significant associations with inadequate treatment response. We recommend a larger sample size that will allow for 1) the estimation of the prevalence of inadequate treatment response, 2) a derivation cohort from which a predictive model could be constructed, and 3) a validation cohort from which a predictive model can be externally validated.

\section{Statement of Authorship}

Both authors participated in the data collection and analysis, and approved the final version submitted.

\section{Author Disclosure}

Both authors declared no conflicts of interest

\section{Funding Source}

This paper was independently funded by the authors.

\section{REFERENCES}

1. Singh JA, Saag KG, Bridges Jr. L, Akl EA, Bannuru RR, Sullivan MC, et al. 2015 American College of Rheumatology guideline for the treatment of rheumatoid arthritis. Arthritis Care Res. 2015; 68(1):1-25. doi:10.1002/acr.22783

2. Penserga E, Natividad TA, Salido E. Clinical profile of 266 Filipino patients with rheumatoid arthritis included in the rheumatoid arthritis database and registry (RADAR) of the Philippine General Hospital. Int J Rheum Dis. 2015; 18(4):433-8. doi:10.1111/1756-185X.12273
3. Cross M, Smith E, Hoy D, Carmona L, Wolfe F, Vos T, et al. The global burden of rheumatoid arthritis: estimates from the Global Burden of Disease 2010 study. Ann Rheum Dis. 2014; 73(7): 1316-22. doi:10.1136/annrheumdis-2013-204627

4. Verstappen SM, Owen SA, Hyrich KL. Prediction of response and adverse events to methotrexate treatment in patients with rheumatoid arthritis. Int J Clin Rheumatol. 2012; 7(5):559-67. doi:10.2217/ ijr.12.57

5. Smolen JS, van Vollenhoven RF, Florentinus S, Chen S, Suboticki JL, Kavanaugh A. Predictors of disease activity and structural progression after treatment with adalimumab plus methotrexate or continued methotrexate monotherapy in patients with early rheumatoid arthritis and suboptimal response to methotrexate. Ann Rheum Dis. 2018; 77(11):1566-72. doi:10.1136/annrheumdis-2018-213502

6. Yu MB, Firek A, Langridge WHR. Predicting methotrexate resistance in rheumatoid arthritis patients. Inflammopharmacology. 2018; 26(3):699-708. doi:10.1007/s10787-018-0459-z

7. Teitsma XM, Jacobs JWG, Welsing PMJ, et al. Inadequate response to treat-to-target methotrexate therapy in patients with newonset rheumatoid arthritis: development and validation of clinical predictors. Ann Rheum Dis. 2018; 77(9):1261-67. doi:10.1136/ annrheumdis-2018-213035

8. Sergeant JC, Hyrich KL, Anderson J, et al. Prediction of primary nonresponse to methotrexate therapy using demographic, clinical and psychosocial variables: Results from the UK Rheumatoid Arthritis Medication Study (RAMS). Arthritis Res Ther. 2018; 20(1):1-11. doi:10.1186/s13075-018-1645-5

9. Drouin J, Haraoui B. Predictors of clinical response and radiographic progression in patients with rheumatoid arthritis treated with methotrexate monotherapy. J Rheumatol. 2010; 37(7):1405-10. doi:10.3899/jrheum

10. Anderson JJ, Wells G, Verhoeven AC, Felson DT. Factors predicting response to treatment in rheumatoid arthritis: the importance of disease duration. Arthritis Rheum. 2000; 43(1):22-9. doi:10.1002/15290131(200001)43:1<22::AID-ANR4>3.0.CO;2-9

11. de Rotte MCFJ, Pluijm SMF, de Jong PHP, Ćalasan MB, Wulffraat $\mathrm{NM}$, Weel AE, et al. Development and validation of a prognostic multivariable model to predict insufficient clinical response to methotrexate in rheumatoid arthritis. PLoS ONE. 2018; 13(12): 1-15. doi:10.1371/journal.pone.0208534

12. Aletaha D, Neogi T, Silman AJ, Funovits J, Felson DT, Bingham CO, et al. 2010 Rheumatoid arthritis classification criteria: An American College of Rheumatology/European League Against Rheumatism collaborative initiative. Arthritis Rheum. 2010; 62(9): 2569-81. doi:10.1002/art.27584 